\title{
EFFECTS OF FLUIDIZATION STATES ON POLARIZATION CHARACTERISTICS OF FLOW-THROUGH PARTICULATE ELECTRODE
}

\author{
MASAO SUDOH, JIRO YUGAMI AND TADASHI SHIROTSUKA \\ Department of Applied Chemistry, Waseda University, Tokyo 160
}

Key Words: Electrolysis, Particulate Electrode, Polarization Curve, Fluidization, Potential Profile

\begin{abstract}
To study the factors influencing the polarization characteristics of a flow-through electrode with electroconductive particles, theoretical analyses have been made by the one-dimensional expression of a two-phase model with combinations of the general electro-chemical reaction rate and the fundamental equation of fluidization state. Potential, overpotential and effectiveness factor of the bed were studied for two systems, fast electrode reaction and slow electrode reaction. To indicate the performance of polarization characteristics of the particulate electrode, bed overpotential was introduced.

Experimentally, potential profiles and polarization curves were measured in the particulate electrode with graphite particles by using the reduction of ferricyanide in alkaline solution. The variation of polarization curves with changing bed expansion were well explained by the calculated results with an available fraction of surface area of electrode of $f=0.38$.
\end{abstract}

\section{Introduction}

The particulate electrode with electrically conductive particles has the major advantages in its large surface area available for electrode reaction and its high mass transfer rate. As total current is expected to be high even at low overpotential, the particulate electrode is suitable for large-volume treatment, i.e., oxidative degradation of organic waste, metal deposition from dilute solution and so on. In the fluidization states of the particles, mass transfer rate increases but unfortunately the effective resistance of the metal phase also increases for poor connection of the inter-particles. Polarization characteristics, i.e., the relation between current density and bed overpotential, is influenced by the fluidization states, the reaction system and the design parameters of the particulate electrode.

Polarization curves have been experimentally measured as a function of the flow rate of the solution. ${ }^{3,10,13)}$ Potential profiles of the porous electrode have been theoretically analysed with a two-phase model by Newman and Tobias, ${ }^{15)}$ and later the model was modified for the fluidized bed electrode. ${ }^{5,8)}$ However, in the discussion of experimental results, electrochemical reaction rate was expressed by an approximate equation in restricted conditions; analytical solutions in the cases of activation control and diffusion control, respectively ${ }^{5,17)}$; a Butler-

\footnotetext{
Received March 4, 1983. Correspondence concerning this article should be addressed to M. Sudoh, now at Dept. of Chem. Eng., Shizuoka University, Hamamatsu 423.
}

Volmer type equation of activation control ${ }^{14)}$; linear expression or power function of overpotential ${ }^{16)}$ and diffusion control equation. ${ }^{1)}$ Goodridge et al. ${ }^{7-9)}$ analysed experiments with the fluidized bed electrodes directly with a non-linear equation obtained by fitting the polarization curve. Kreysa ${ }^{11}$ introduced a rate equation characterized by combined diffusion and activation control, which had the simplification of an irreversible process far from equilibrium.

In the previous work, ${ }^{19)}$ the mixed potential of the photogalvanic cell with flow-through electrodes was analysed by using the general expression of electrochemical reaction rate on the two-phase model without resistivity in the metal phase.

In this paper, theoretical analyses of the characteristics of the particulate electrode are made with combinations of the general equation of electrode reaction and the design parameters of the fluidization state. The potential profile and the polarization curve in the particulate electrode of graphite are measured experimentally with the reduction of ferricyanide.

\section{Theoretical}

\subsection{Fundamental equation}

The reactant solution flows through the bed of particles on the surface where the electrode reaction occurs. For simplification, the concentration change in the bulk solution is ignored in the bed. The electrochemical reaction rate is generally expressed by the Butler-Volmer equation, including the mass transfer step. ${ }^{19)}$ 


$$
i=\frac{i_{0}\left\{\exp \left(\beta_{1} \eta\right)-\exp \left(-\beta_{2} \eta\right)\right\}}{1+\delta_{R} \exp \left(\beta_{1} \eta\right)+\delta_{0} \exp \left(-\beta_{2} \eta\right)}
$$

where $\beta_{1}=\alpha_{a} n \boldsymbol{F} / \boldsymbol{R} T, \quad \beta_{2}=\alpha_{c} n \boldsymbol{F} / \boldsymbol{R} T, \quad \delta_{R}=i_{0} / n \boldsymbol{F} k_{R} C_{R}$ and $\delta_{O}=i_{0} / n F k_{O} C_{O}$. Now, $\beta_{1}, \beta_{2}, \delta_{R}$ and $\delta_{O}$ are constant values independent of $x$ and time.

Based on the one-dimensional two-phase model of the particulate electrode, current densities in the metal (particulate) phase and the solution phase $i_{m}$ and $i_{s}$, respectively, are given as follows.

$$
\begin{aligned}
& i_{m}=-\left(1 / \rho_{m}\right)\left(\mathrm{d} \Phi_{m} / \mathrm{d} x\right) \\
& i_{s}=-\left(1 / \rho_{s}\right)\left(\mathrm{d} \Phi_{s} / \mathrm{d} x\right)
\end{aligned}
$$

In consideration of the cathodic reaction, the current balance in the bed is as follows.

$$
\mathrm{d} i_{m} / \mathrm{d} x=-a i
$$

Then the current density per unit cross-sectional area of the bed, $i$, is the total of the current densities of the metal phase, $i_{m}$, and the solution phase, $i_{s}$, and is assumed to be independent of $x$.

$$
i=i_{m}+i_{s}
$$

Overpotential, $\eta$, is defined as the difference of electrode potential, $\left(\Phi_{m}-\Phi_{s}\right)$, and equilibrium potential $E_{e}$.

$$
\eta=\Phi_{m}-\Phi_{s}-E_{e}
$$

By arranging Eqs. (2)-(6),

$$
d^{2} \eta / \mathrm{d} x^{2}=\left(\rho_{m}+\rho_{s}\right) a i
$$

Boundary conditions are as follows.

$$
\begin{array}{ll}
i_{s}=0, & i_{m}=\boldsymbol{i}, \quad \eta=\eta_{0}, \text { at } \quad x=0 \\
i_{s}=i, & i_{m}=0 \quad \text { at } x=L
\end{array}
$$

Then $\eta, i, i_{m}$ and $i_{s}$ are dependent on $x$. Using Eqs. (2), (3), and (5), the following relation is obtained.

$$
\mathrm{d} \eta / \mathrm{d} x=-\rho_{m} i_{m}+\rho_{s} i_{s}
$$

The boundary conditions are replaced by substituting Eqs. (8) and (9) into Eq. (10).

$$
\begin{aligned}
& \mathrm{d} \eta / \mathrm{d} x=-\rho_{m} i, \quad \eta=\eta_{0} \quad \text { at } \quad x=0 \\
& \mathrm{~d} \eta / \mathrm{d} x=\rho_{s} i \quad \text { at } \quad x=L
\end{aligned}
$$

Finally,

$$
i=\int_{0}^{L} a i \mathrm{~d} x
$$

\subsection{Calculation procedure}

At an assumed value of $i$, numerical calculation of Eq. (7) with Eq. (1) is made by the Range-Kutta-Gill method. The calculation is repeated until the calculated value of $\eta$ agrees with the assumed value from Eqs. (11) and (12). With the determined value of $i, i_{m}$ is calculated by Eqs. (4) and (8). At the same time. $\Phi_{m}$ and $\Phi_{s}$ are obtained from Eqs. (2), (3) and (5).

\subsection{Calculated results}

Reaction systems are divided into two types: fast electrode reaction with $i_{0}=1.0 \mathrm{~A} \cdot \mathrm{m}^{-2}$ (abbreviated FRS) and slow electrode reaction with $i_{0}=$ $10^{-3} \mathrm{~A} \cdot \mathrm{m}^{-2}$ (SRS). The limiting current density, $i_{L}$, is given from Eqs. (1) and (13) as

$$
-i_{L}=a i L / \delta_{0}
$$

Calculation was made under the conditions of $-i_{L}=2 \times 10^{2} \mathrm{~A} \cdot \mathrm{m}^{-2}, \quad a=5 \times 10^{3} \mathrm{~m}^{-1}, \quad \alpha_{a}=\alpha_{c}=0.5$, $n=1, \quad T=298 \mathrm{~K}, \quad L=2 \times 10^{-2} \mathrm{~m} \quad$ and $\quad \beta_{1}=\beta_{2}=$ $19.47 \mathrm{~V}^{-1}$. By changing the values of $i_{0}, \rho_{m} / \rho_{s}$ and $\eta_{0}$, the overpotential and potential profiles were calculated.

\subsubsection{Overpotential profile at $\rho_{m}=0$}

Figure 1 shows the profiles of dimensionless overpotentials. In FRS, the overpotential has the predominant distribution at the lower values of $\left(-\eta_{0}\right)$ and the value of $(-\eta) /\left(-\eta_{0}\right)$ is large at the position of $x / L=1$. In SRS, the overpotential has a relatively flat profile at low values of $\left(-\eta_{0}\right)$ and shows the similar curve to that of FRS at high values of $\left(-\eta_{0}\right)$.

\subsubsection{Effects of $\rho_{m} / \rho_{s}$ ratio}

Figure 2 shows the profiles of the metal and the solution potentials and overpotential as a function of $\rho_{m} / \rho_{s}$ of FRS. At larger values of $\rho_{m} / \rho_{s}$, the overpotential has lower values in the bed except in the zone near the feeder electrode. Then, to estimate the fraction available for the electrode reaction of the particles in the bed, the effectiveness factor, a modified type of that of Paulin et al., ${ }^{16)}$ is introduced.

$$
\begin{aligned}
E & =\frac{\text { actual current density }}{\begin{array}{c}
\text { current density obtained at the maximum } \\
\text { value of overpotential in total bed }
\end{array}} \\
& =\frac{i}{\int_{0}^{L} \text { ai }\left(\eta_{\max }\right) \mathrm{d} x}
\end{aligned}
$$

Figure 3 shows the relations between the effectiveness factor, $E$, and the overpotential at feeder electrode, $\left(-\eta_{0}\right)$. In FRS, as $\left(-\eta_{0}\right)$ increases, $E$ increases and reaches the maximum value, 1 . In SRS, $E$ exhibits the minimum value at $\left(-\eta_{0}\right) \fallingdotseq 0.4 \mathrm{~V}$. As the ratio of $\rho_{m}$ to $\rho_{s}$ becomes large, $E$ decreases and has the value of 1 under extremely restricted conditions of very low or very high overpotential.

\subsubsection{Bed overpotential}

On the plate electrode, overpotential is expressed by Eq. (6) and the performance of the electrode is discussed by the relations between $i$ and $\eta$. Bed overpotential of the particulate electrode is defined by the next equation. 


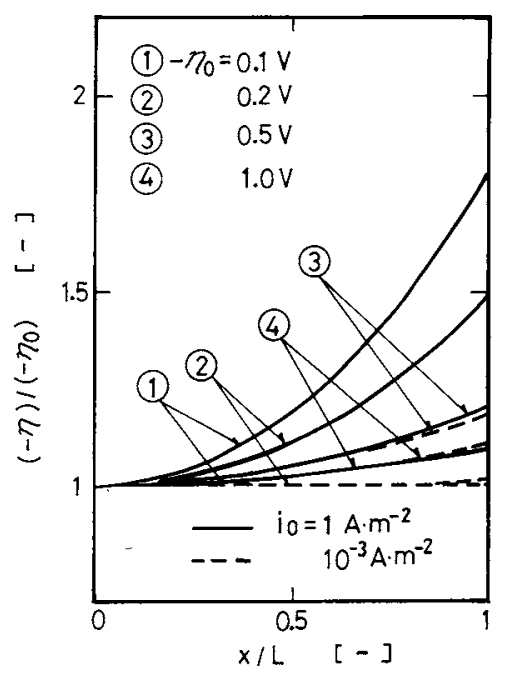

Fig. 1. Dimensionless overpotential profiles with different inlet overpotentials at $\rho_{m}=0$.

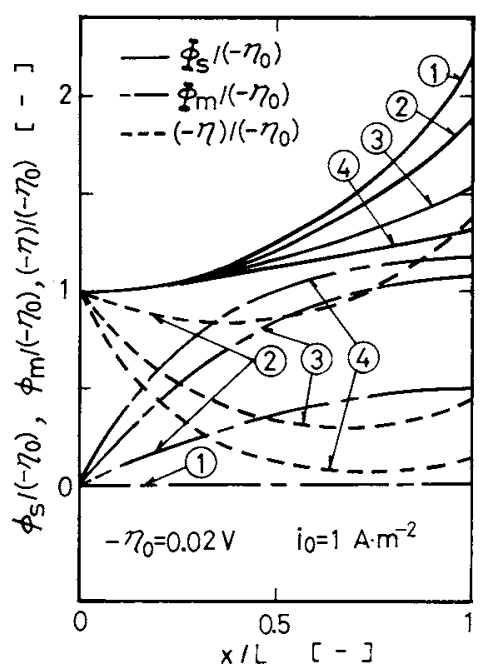

Fig. 2. Dimensionless potential and overpotential with different $\rho_{m} / \rho_{s}$ ratios; $\rho_{m} / \rho_{s}$ is 0 (1)), 0.5 ((2)), 3.0 (3) and 9.0 (4)).

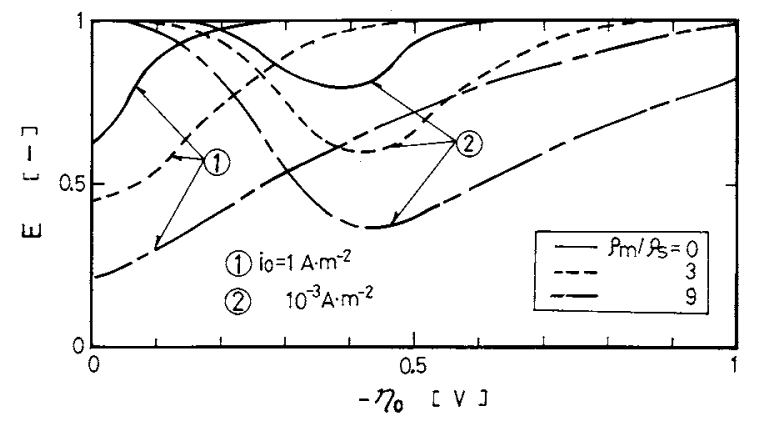

Fig. 3. Relations between effectiveness factor and inlet overpotential with different $\rho_{m} / \rho_{s}$ ratios.

$$
\eta_{\mathrm{bed}}=\Phi_{m}(0)-\Phi_{s}(L)-E_{e}
$$

Then,

$$
\begin{aligned}
-\eta_{\text {bed }} & =-\eta_{0}+\left\{\Phi_{s}(L)-\Phi_{s}(0)\right\} \\
& =-\eta_{L}+\left\{\Phi_{m}(L)-\Phi_{m}(0)\right\}
\end{aligned}
$$

At $\rho_{m}=0$, the next equation holds.

$$
-\eta_{\text {bed }}=-\eta_{L}
$$

When the reaction is governed by mass transfer rate at the larger negative value of overpotential, electrochemical reaction rate is as follows.

$$
i=-i_{0} \delta_{0}^{-1}
$$

By substituting Eq. (19) into Eq. (7) and integrating Eq. (7) with the boundary conditions of Eqs. (11) and (12), overpotential at $x=L,\left(-\eta_{L}\right)$, is obtained.

$$
-\eta_{L}=-\eta_{0}+\left(\rho_{s}-\rho_{m}\right)\left(-\boldsymbol{i}_{L}\right) L / 2
$$

Then, at $\rho_{m}=0$,

$$
-\eta_{\text {bed }}=-\eta_{0}+\rho_{s}\left(-i_{L}\right) L / 2
$$

At $\rho_{m} \neq 0$, the next relation is determined.

$$
-\eta_{\text {bed }}\left(\text { at } \rho_{m} \neq\right) \leq-\eta_{\text {bed }}\left(\text { at } \rho_{m}=0\right)
$$

In the present calculation, the value of $\rho_{s}\left(-\boldsymbol{i}_{L}\right) L / 2$ is obtained as $0.1 \mathrm{~V}$. In Fig. 4, as the ratio of $\rho_{\mathrm{m}} / \rho_{\mathrm{s}}$ increases, $\left(-\eta_{\text {bed }}\right)$ reaches the value of Eq. (21) at higher values of $\left(-\eta_{0}\right)$.

\subsection{Design parameters of fluidization of particles}

Design parameters are obtained by certain values of bed expansion, $E x$, diameter of particle, $d_{p}$, intrinsic specific resistance of electrolyte, $\rho_{s, 0}$, liquid holdup and liquid velocity at minimum fluidization, $\varepsilon_{\mathrm{mf}}$ and $u_{\mathrm{mf}}$, respectively. Liquid holdup is as follows.

$$
\varepsilon=1-\left(1-\varepsilon_{\mathrm{mf}}\right) / E x
$$

Specific surface area of particles available for electrode reaction is modified by the factor, $f$, of the geometrical value of specific surface area.

$$
a=6 f(1-\varepsilon) / d_{p}
$$

Mass transfer coefficients are calculated by Shirai's equation ${ }^{18)}$ of Eq. (25) with the liquid velocity of Eq. (26).

$$
\begin{gathered}
\varepsilon\left(k d_{p} / D\right)=2.0+0.75\left(d_{p} u \rho_{F} / \mu_{F}\right)^{1 / 2}\left(\mu_{F} / \rho_{F} D\right)^{1 / 3} \\
u=u_{\mathrm{mf}}\left(1-\varepsilon_{\mathrm{mf}}\right) \varepsilon^{3} /\left\{(1-\varepsilon) \varepsilon_{\mathrm{mf}}^{3}\right\}
\end{gathered}
$$

Effective specific resistances of the solution phase and the metal phase are calculated by Bruggeman's equation $^{4)}$ and Kreysa's relationship, ${ }^{12)}$ respectively.

$$
\begin{aligned}
& \rho_{s}=\rho_{s, 0}^{-1.5} \\
& \rho_{m}=E x^{1 / 3}\left(E x^{1 / 3}-1\right)(1-\varepsilon) \varepsilon /\left(2 \pi u C_{F}\right)
\end{aligned}
$$

1.5 Effects of fluidization states on polarization characteristics

Calculation conditions are $d_{p}=10^{-3} \mathrm{~m}, f=1, \varepsilon_{\mathrm{mf}}=$ $0.52, u_{\mathrm{mf}}=2.1 \times 10^{-3} \mathrm{~m} \cdot \mathrm{s}^{-1}, C_{R}=C_{O}=1.0 \mathrm{~mol} \cdot \mathrm{m}^{-3}$, $\rho_{F}=1.0 \times 10^{3} \mathrm{~kg} \cdot \mathrm{m}^{-3}, \quad \mu_{F}=1.2 \times 10^{-3} \mathrm{~kg} \cdot \mathrm{m}^{-1} \cdot \mathrm{s}^{-1}$, $D_{R}=D_{O}=1.0 \times 10^{-9} \mathrm{~m}^{2} \cdot \mathrm{s}^{-1}, \quad C_{F}=1.0 \mathrm{~F} \cdot \mathrm{m}^{-2}$ and $\rho_{s, 0}=5.0 \times 10^{-2} \Omega \cdot \mathrm{m}$ in addition to the above- 


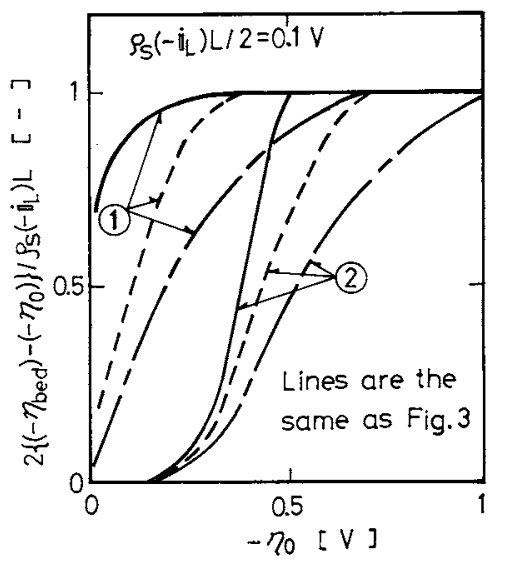

Fig. 4. Relations between bed overpotential and inlet overpotential.

mentioned conditions.

Relations between bed potential and current density are shown in Figs. 5 and 6 . In the fixed bed ( $E x=1, u \leq u_{\mathrm{mf}}$ ), current density is highest at $u=u_{\mathrm{mf}}$ at every bed overpotential. In the fluidized bed $(E x>1$, $u>u_{\mathrm{mf}}$ ), current density is lower at low values of bed overpotential than that in the fixed bed but higher at high values. These phenomena are predominant in FRS.

\section{Experimental Apparatus and Procedure}

Experimental apparatus is a vertical cylinder of polyacrylate resin (I.D. $25 \mathrm{~mm} \phi$ ), schematically shown in Fig. 7. Feeder electrode and electroconductive particles are made from graphite. The graphite particles were prepared by collecting the sieved fraction of $0.59-0.71 \mathrm{~mm}$ diameter of crushed graphite. The particles have irregular shape of mean diameter of $0.65 \mathrm{~mm}$. Reference electrode is $\mathrm{Ag} / \mathrm{AgCl}$ (saturated $\mathrm{KCl}$ solution). Inlet overpotential at the feeder electrode was controlled by a potentiostat (HS-201, Hokuto Denko) with a Luggin capillary. Potentials of the metal and the solution phase were measured by the composite probe shown in Fig. 7. Electrode reaction is the reduction of ferricyanide in alkaline solution of $1.0 \times 10^{3} \mathrm{~mol} \cdot \mathrm{m}^{-3} \mathrm{NaOH}$. The solution flows down from head tank to particulate electrode and recycles to sub-tank and to head tank blanketed by nitrogen gas. All parts except the particulate electrode were covered with black tapes for protection from photodecomposition. Experimental conditions are shown in Table 1.

\section{Experimental Results and Discussion}

The measured profiles of potentials are shown in Fig. 8. Overpotential obtained by Eq. (6) is shown with broken lines. One-dotted lines indicate the calculated values below. $\rho_{m}$ and $\rho_{s}$ in Fig. 9 were calculated by Eqs. (11) and (12). In the fixed bed $(E x=1), \rho_{m}$ and

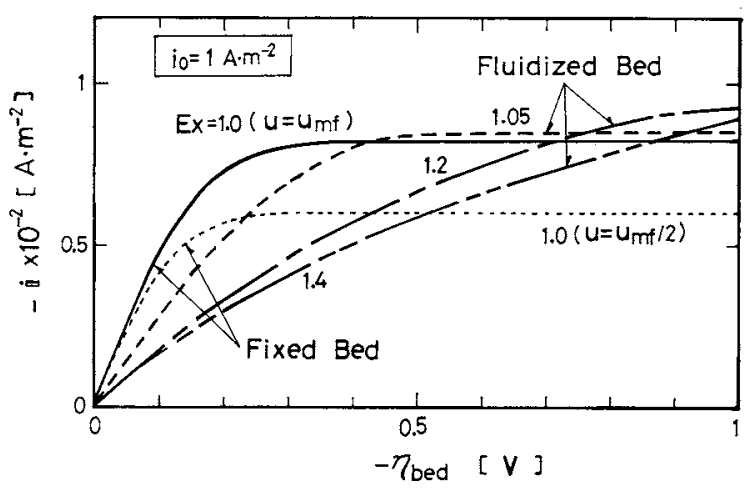

Fig. 5. Polarization curves of model calculation with different bed expansions for $i_{0}=1.0 \mathrm{~A} \cdot \mathrm{m}^{-2}$.

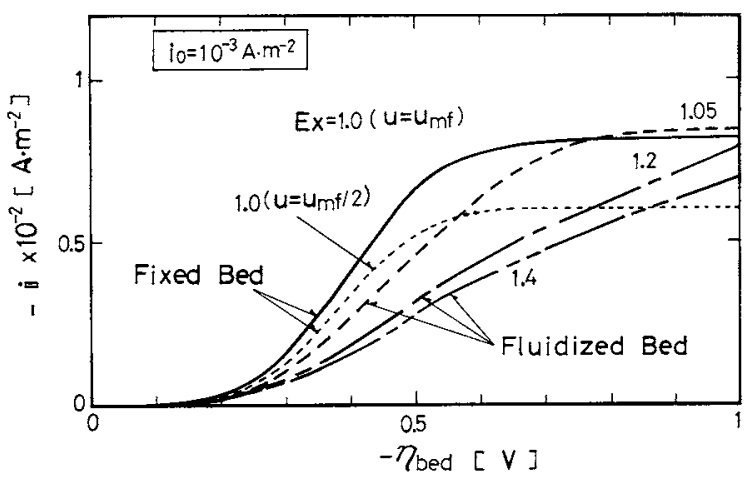

Fig. 6. Polarization curves of model calculation with different bed expansions for $i_{0}=10^{-3} \mathrm{~A} \cdot \mathrm{m}^{-2}$.

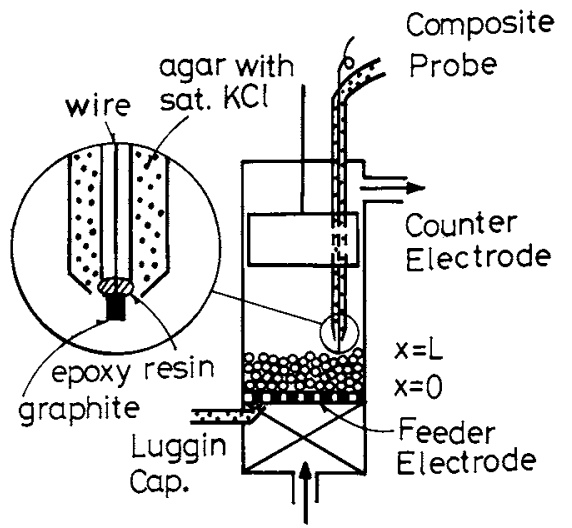

Fig. 7. Schematic diagram of flow-through particulate electrode.

Table 1. Experimental conditions

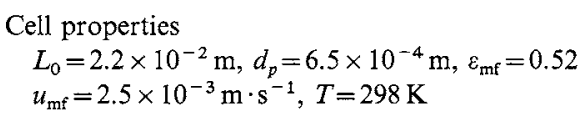

$\begin{array}{ll}\text { Solution properties } & \text { Reaction properties } \\ C_{R}=C_{o}=1.0 \mathrm{~mol} \cdot \mathrm{m}^{-3} & i_{0}=3.0 \times 10^{-1} \mathrm{~A} \cdot \mathrm{m}^{-2 *} \\ \rho_{s, 0}=5.8 \times 10^{-2} \Omega \cdot \mathrm{m}^{*} & \alpha_{c}=0.46^{*} \\ D_{R}=6.00 \times 10^{-10} \mathrm{~m}^{2} \cdot \mathrm{s}^{-1} & \alpha_{a}=0.54^{*} \\ D_{o}=5.17 \times 10^{-10} \mathrm{~m}^{2} \cdot \mathrm{s}^{-1} & n=1 \\ \rho_{F}=1.0 \times 10^{3} \mathrm{~kg} \cdot \mathrm{m}^{-3} & E_{e}=0.25 \mathrm{~V} \\ \mu_{F}=1.4 \times 10^{-3} \mathrm{~kg} \cdot \mathrm{m}^{-1} \cdot \mathrm{s}^{-1} & \end{array}$

*. Values obtained by preliminary experiments. 

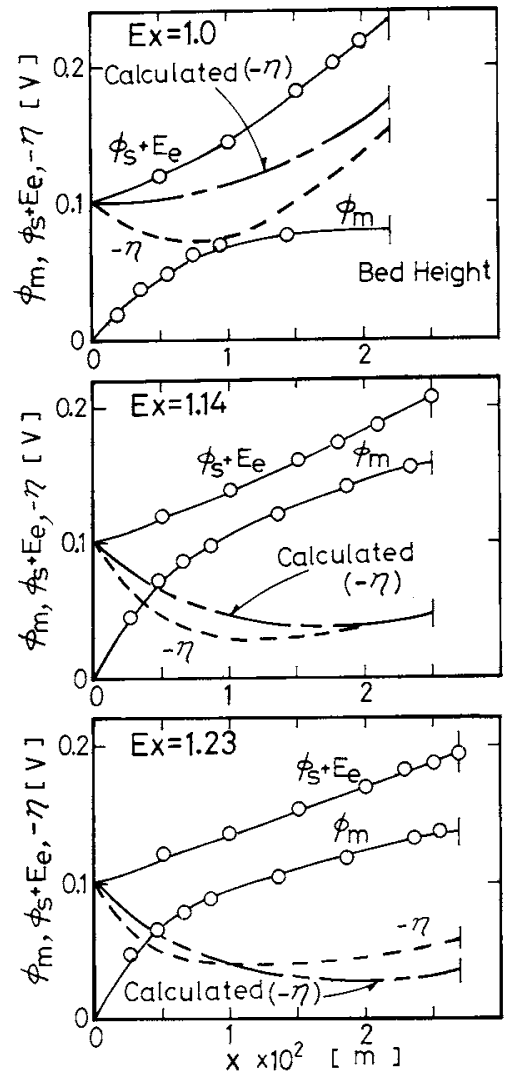

Fig. 8. Experimental results of potentials and overpotentials at $E x=1,1.14$ and 1.23 .

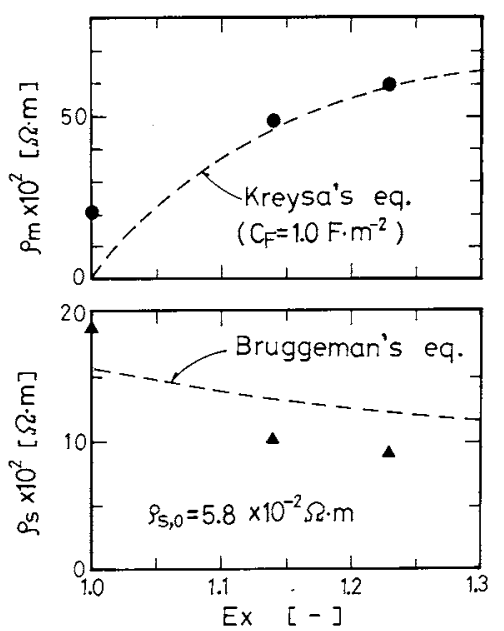

Fig. 9. Experimental results of $\rho_{m}$ and $\rho_{s}$ calculated from overpotential profiles.

$\rho_{s}$ are larger than the calculated values. It is understood that graphite grains have high contact resistance in the fixed bed. $\rho_{m}$ is well explained by Eq. (28) with the double-layer capacity of $C_{F}=$ $1.0 \mathrm{~F} \cdot \mathrm{m}^{-2}$. This value agrees well with the measured value for porous graphite by Brown et al. ${ }^{2)}$

Figure 10 shows the relations between bed overpotential and current density with different bed expansion. Due to the irregular shape of the particles,

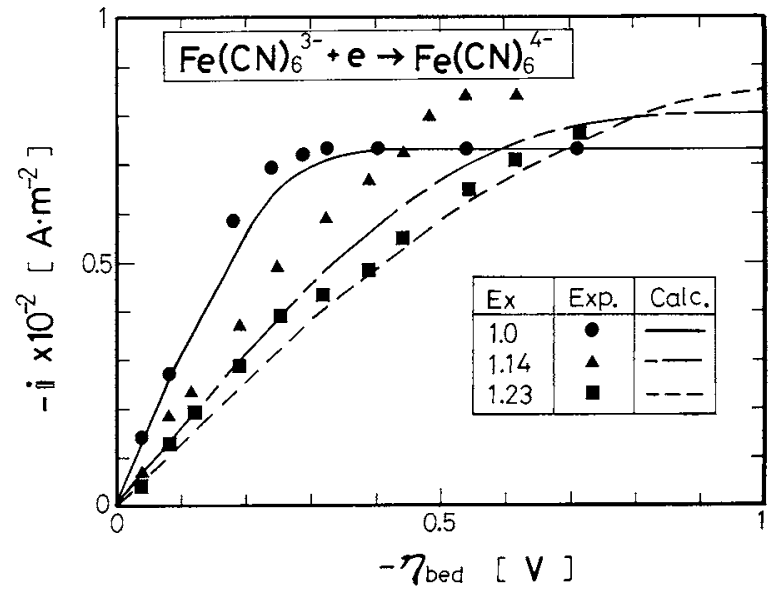

Fig. 10. Comparison between experimental and theoretical polarization curves with different bed expansions.

the contact area between adjacent particles is assumed to be large. This would tend to reduce the available fraction of surface area of electrode, $f$. Current density was calculated as a function of $f$ using the same method as in Section 1.5 with the experimental conditions shown in Table 1 and $\rho_{s}$ of Eq. (27) and $\rho_{m}$ of Eq. (28), assuming that $C_{F}=1.0 \mathrm{~F} \cdot \mathrm{m}^{-2}$. Then $f$ was given as 0.38 by fitting the calculated value with the experimental one of limiting current density of the fixed bed $(E x=1)$. Also, the calculated relations between $(-\boldsymbol{i})$ and $\left(-\eta_{\text {bed }}\right)$ agree relatively well with the experimental ones. It was reported that $f=0.6$ $1.0^{8)}$ in a fluidized bed with platinum-coated glass beads and that $f=0.5^{20)}$ in a fluidized bed with graphite grains. Experimental results were relatively higher than the calculated ones at $E x>1$. It is inferred that the available fraction of surface area is larger in the fluidized bed than that in the fixed bed. Surface area available for electrode reaction and the double-layer capacity are subjects of further studies in the future.

\section{Conclusion}

To study the characteristics of the flow-through particulate electrode, theoretical analyses were made with the general equation of electrochemical reaction rate and the fundamental equation of fluidization state. Effects of reaction rate were studied for two systems, fast electrode reaction (FRS) and slow electrode reaction (SRS). Potential, overpotential and effectiveness factor of the bed were analysed as a function of $\rho_{m} / \rho_{s},\left(-\eta_{0}\right)$ and $i_{0}$. In particular, effectiveness factor showed different behavior for FRS and SRS.

Bed overpotential was introduced for representing the performance of the polarization characteristics of the particulate electrode and Eqs. (20), (21) were proposed. The relation between bed overpotential and current density was discussed with bed expansion 
in the fluidization state.

In experiments with the reduction of ferricyanide, $\rho_{m}$ and $\rho_{s}$ were calculated by overpotential profile. The polarization curves were well explained by the calculated results with an available fraction of surface area of electrode of $f=0.38$.

\begin{tabular}{|c|c|c|}
\hline \multicolumn{3}{|c|}{ Nomenclature } \\
\hline$a$ & $\begin{aligned}= & \text { specific surface area available for electr } \\
& \text { reaction }\end{aligned}$ & rode $\left[\mathrm{m}^{-1}\right]$ \\
\hline$C$ & $=$ concentration & {$\left[\mathrm{mol} \cdot \mathrm{m}^{-3}\right]$} \\
\hline$C_{F}$ & $=$ double-layer capacity & {$\left[\mathrm{F} \cdot \mathrm{m}^{-2}\right]$} \\
\hline$D$ & $=$ diffusion coefficient & {$\left[\mathrm{m}^{2} \cdot \mathrm{s}^{-1}\right]$} \\
\hline$d_{p}$ & $=$ diameter of particle & {$[\mathrm{m}]$} \\
\hline$E$ & $\begin{array}{l}=\text { effectiveness factor of bed defined by } \\
\text { Eq. (15) }\end{array}$ & {$[-]$} \\
\hline$E_{e}$ & $=$ equilibrium potential & {$[\mathrm{V}]$} \\
\hline Ex & $=$ bed expansion & {$[-]$} \\
\hline $\boldsymbol{F}$ & $=$ Faraday's constant & {$\left[\mathrm{C} \cdot\right.$ eq. $\left.^{-1}\right]$} \\
\hline$f$ & $\begin{aligned}= & \text { electroactive fraction of geometrical su } \\
& \text { area of particles }\end{aligned}$ & Irface $[-]$ \\
\hline$i$ & $=$ current density of bed & {$\left[\mathrm{A} \cdot \mathrm{m}^{-2}\right]$} \\
\hline$i$ & $=$ current density & {$\left[\mathrm{A} \cdot \mathrm{m}^{-2}\right]$} \\
\hline$i_{0}$ & $=$ exchange current density & {$\left[\mathrm{A} \cdot \mathrm{m}^{-2}\right]$} \\
\hline$i_{L}$ & $=$ limiting current density & {$\left[\mathrm{A} \cdot \mathrm{m}^{-2}\right]$} \\
\hline$k$ & $=$ mass transfer coefficient & {$\left[\mathrm{m} \cdot \mathrm{s}^{-1}\right]$} \\
\hline$L$ & $=$ bed height & {$[\mathrm{m}]$} \\
\hline$L_{0}$ & $=$ bed height of fixed bed & {$[\mathrm{m}]$} \\
\hline $\boldsymbol{R}$ & $=$ gas constant & $\left.\mathrm{K}^{-1} \cdot \mathrm{mol}^{-1}\right]$ \\
\hline$T$ & $=$ temperature & {$[\mathrm{K}]$} \\
\hline$u$ & $=$ liquid velocity & {$\left[\mathrm{m} \cdot \mathrm{s}^{-1}\right]$} \\
\hline$u_{\mathrm{mf}}$ & $=$ minimum fluidization velocity & {$\left[\mathrm{m} \cdot \mathrm{s}^{-1}\right]$} \\
\hline$x$ & $=$ bed height coordinate & {$[\mathrm{m}]$} \\
\hline$\alpha$ & $=$ apparent transfer coefficient & {$[-]$} \\
\hline$\beta_{1}, \beta_{2}$ & $=$ constants used in Eq. (1) & {$\left[V^{-1}\right]$} \\
\hline$\delta$ & $=$ constant used in Eq. (1) & {$[-]$} \\
\hline$\varepsilon$ & $=$ liquid holdup & {$[-]$} \\
\hline$\varepsilon_{m f}$ & $=$ liquid holdup at minimum fluidization & {$[-]$} \\
\hline$\Phi$ & $=$ potential & [V] \\
\hline$\eta$ & $=$ overpotential & [V] \\
\hline$\mu_{F}$ & $=$ viscosity of solution & $\left.\mathrm{g} \cdot \mathrm{m}^{-1} \cdot \mathrm{s}^{-1}\right]$ \\
\hline$\rho$ & $=$ effective specific resistance & {$[\Omega \cdot \mathrm{m}]$} \\
\hline$\rho_{\mathrm{s}, 0}$ & $\begin{aligned}= & \text { intrinsic specific resistance of solution } \\
& \text { phase without particles }\end{aligned}$ & {$[\Omega \cdot \mathrm{m}]$} \\
\hline$\rho_{F}$ & $=$ density of solution & {$\left[\mathrm{kg} \cdot \mathrm{m}^{-3}\right]$} \\
\hline
\end{tabular}

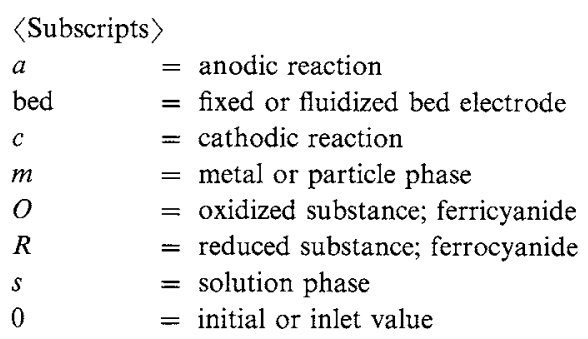

\section{Literature Cited}

1) Bennion, D. N. and J. Newman: J. Appl. Electrochem., 2, 113 (1972).

2) Brown, G. M. and F. A. Posey: J. Electrochem. Soc., 128, 306 (1981).

3) Buckhurst, J. R., J. M. Coulson, F. Goodridge and R. E. Plimley: J. Electrochem. Soc., 116, 1600 (1969).

4) De La Rue, R. E. and C. W. Tobias: J. Electrochem. Soc., 106, 827 (1959).

5) Fleischman, M. and J. W. Oldfield: J. Electroanal. Chem., 29, $211(1971)$

6) Fleischman, M., J. W. Oldfield and D. F. Porter: $J$. Electroanal. Chem., 29, 241 (1971).

7) Germain, S. and F. Goodridge: Electrochim. Acta, 21, 545 (1976)

8) Goodridge, F., D. I. Holden, M. D. Murray and R. F, Plimley: Trans. Inst. Chem. Eng., 49, 128 (1971).

9) Goodridge, F. and B. M. Ismail: Inst. Chem. Engrs., Symp. Ser., 1, No. 37, p. 29 (1971).

10) Hiddleston, J. N. and A. F. Douglas: Electrochim. Acta, 15, 431 (1970).

11) Kreysa, G.: Electrochim. Acta, 23, 1351 (1978)

12) Kreysa, G.: Electrochim. Acta, 25, 813 (1980).

13) Kreysa, G., S. Pionteck and E. Heitz: J. Appl. Electrochem., 5, 305 (1975).

14) Kusakabe, K., S. Morooka and Y. Kato: J. Chem. Eng. Japan, 14, 208 (1981).

15) Newman, J. S. and C. W. Tobias: J. Electrochem. Soc., 109 , 1183 (1962).

16) Paulin, M., D. Hutin and F. Coeuret: J. Electrochem. Soc., 124, 180 (1977).

17) Pickett, D. J.: J. Appl. Electrochem., 5, 95 (1977).

18) Shirai, T.: "Ryudoso," Kagaku Gijutsusha, p. 207 (1964).

19) Sudoh, M., T. Murakami and T. Shirotsuka: J. Chem. Eng. Japan, 16, 61 (1983).

20) Wegner, R. S. and D. N. Bennion: J. Appl. Electrochem., 6, 385 (1976). 